EPJ Web of Conferences 71, 00014 (2014)

DOI: 10.1051/epjconf/20147100014

(C) Owned by the authors, published by EDP Sciences, 2014

\title{
Measurements of diboson production with the ATLAS detector
}

\author{
Federico Bertolucci ${ }^{1}$,a on behalf of the ATLAS Collaboration \\ ${ }^{1}$ INFN-Sezione di Pisa and University of Pisa
}

\begin{abstract}
.
The study of the diboson production characteristics with the ATLAS detector at the Large Hadron Collider in proton-proton collisions at $\sqrt{s}$ up to $8 \mathrm{TeV}$ allows to measure the behaviour of the electroweak interactions in an unexplored energy range. This measurement allows to test the Standard Model theory and to search for new Physics by probing possible anomalous couplings at the gauge-boson vertices. This studies are also important because the Standard Model $W^{+} W^{-}$and $Z Z$ productions are irreducible backgrounds for searches of the Higgs boson in the diboson decay channels.

The measurements of diboson production in ATLAS are presented for different electroweak diboson channels: $Z Z, W Z, W W, W \gamma$ and $Z \gamma$. Anomalous triple-gauge coupling limits that are obtained from these measurements are also presented.
\end{abstract}

\section{Introduction}

The production of pairs of bosons at the Large Hadron Collider (LHC) in proton-proton collisions provides the opportunity to test the electroweak sector of the Standard Model (SM) at the TeV scale. The interactions between bosons are particularly interesting as they test the self-couplings as predicted by the non-Abelian gauge symmetry of the electroweak sector (see [1] for a review); deviations from the SM predictions could be indicative of the production of new resonances decaying into bosons or of other non-SM contributions and scenarios; furthermore, diboson final states are one of the irreducible SM backgrounds to the Higgs boson detection.

A review of measurements of diboson final states at proton-proton collisions at the LHC with the ATLAS detector is given, reporting results obtained with interactions at $\sqrt{s}=7 \mathrm{TeV}$ and $8 \mathrm{TeV}$.

\section{Gauge couplings}

The vector boson self-interactions are fundamental and successful predictions of the Standard Model of particle interactions. The non-abelian nature of the Electroweak symmetry allows for couplings among bosons, but some of them are forbidden by other symmetries and invariances. The possibility to study diboson final states sets limits to the variations of the coupling strenghts and to the nonallowed couplings, the anomalous Triple and Quartic Gauge Couplings (aTGCs and aQGCs); this report focuses only on aTGC results. The aTGC parameters are expected to be zero in the Standard Model scenario: if some of them are not zero, their effect is to modify the cross sections and the

a e-mail: federico.bertolucci@pi.infn.it 
kinematics of the diboson decay products, in particular at high transverse momentum of the bosons associated to a vertex in the theory. In particular, the total neutral TGCs ( $Z Z Z, Z Z \gamma$ and $Z \gamma \gamma)$ are not allowed in the Standard Model. The usual approach is to express the SM Lagrangian as an effective model independent theory in which TGCs appears as parameters. Limits on these parameters are set using symmetry and experimental results.

The diboson final states have been investigated in experiments at LEP [2] and Tevatron [3], however the triple gauge boson couplings have not yet been determined with the same precision as the boson couplings to fermions.

In the following Sections the ATLAS detector (Section 3), the object definitions (Section 4) and the common aspects of the various diboson final state measurements are presented (Section 5); the measurements are described in Sections from 6 to 11. In Section 12 the results obtained with the presented measurements are collected.

\section{ATLAS detector and LHC experimental conditions}

The ATLAS detector is a multi-purpose system situated along the LHC. It has a cylindrical symmetry along the beam axis, and consists of an Inner Detector for charged tracking, surrounded by electromagnetic and hadronic calorimeters, and finally a muon detector system.

The Inner Detector is immersed in a superconducting $2 \mathrm{~T}$ axial magnetic field solenoid, and it consists of three subsystems: the pixel and silicon strips detectors cover the pseudorapidity ${ }^{1}$ range $|\eta|<2.5$, while the straw tubes of the Transition Radiation Tubes cover the region up to $|\eta|<2$. The highly segmented electromagnetic calorimeter plays a crucial role in electron and photon identification and uses lead as absorber and liquid Argon as active material, covering the region $|\eta|<3.2$; the tile hadronic calorimeter is a steel/scintillating-tile detector and it is situated outside the envelope of the electromagnetic calorimeter; the calorimeter coverage is extended up to $|\eta|<4.9$ by endcaps and forward calorimeters. The muon spectrometer is based on large superconducting aircore toroid magnets, three stations of chambers assure precise muon track measurements in the range $|\eta|<2.4$. The detector and its performance are described in detail in [4].

Table 1. Data-taking conditions of the ATLAS experiment for the two different scenarios used for the presented measurements.

\begin{tabular}{|c||c|c|c|}
\hline & $<\mu>$ & peak luminosity $\left(\mathrm{cm}^{-2} \mathrm{~s}^{-1}\right)$ & delivered data $\left(\mathrm{fb}^{-1}\right)$ \\
\hline \hline$\sqrt{s}=7 \mathrm{TeV}$ & 9.1 & $\sim 4 \cdot 10^{33}$ & $5.46(4.57$ good for Physics $)$ \\
$\sqrt{s}=8 \mathrm{TeV}$ & 20.7 & $\sim 7.7 \cdot 10^{33}$ & $22.8(20.3$ good for Physics $)$ \\
\hline
\end{tabular}

The presented results have been obtained with data acquired from proton-proton collisions at two center of mass energies, $\sqrt{s}=7 \mathrm{TeV}$ and $8 \mathrm{TeV}$. Table 1 summarizes the center-of-mass energy, pileup conditions $(<\mu>$ is the average number of interactions per bunch crossing) and total amount of data available for the analyses ([5] and [6]).

\footnotetext{
${ }^{1}$ ATLAS uses a right-handed coordinate system with its origin at the nominal interaction point. The $z$-axis is along the beam pipe, the $x$-axis points to the centre of the LHC ring and the $y$-axis points upward. Cylindrical coordinates $(r, \phi)$ are used in the transverse plane, $\phi$ being the azimuthal angle around the beam pipe. The pseudorapidity $\eta$ is defined as $\eta=-\ln [\tan (\theta / 2)]$, where $\theta$ is the polar angle. The angular distance between two vectors is defined as sum in quadrature of the separation in azimuth and pseudorapidity, $\Delta R=\sqrt{(\Delta \phi)^{2}+(\Delta \eta)^{2}}$. The transverse momentum $p_{T}$ is the component of the momentum measured on the transverse plane.
} 


\section{Physics Objects definition}

In all the presented analyses, the $W$ and $Z$ bosons are reconstructed from their decay products, triggering the events on the presence of a lepton of a given $p_{T}$ threshold. Selections are usually applied on lepton and jet reconstruction quality, multiplicity, angular requirements, as well as on the missing transverse energy $\left(E_{T}^{\text {miss }}\right)$ and event overall quality. The corresponding physical objects are defined in ATLAS as follows:

- electrons: a candidate electron is obtained from an energy cluster in the EM calorimeter, providing the particle energy, associated with a reconstructed track in the ID, which gives information on the direction; the electrons are usually requested to be isolated both using tracks and calorimetric informations;

- muons: muons are reconstructed matching the tracks from the Muon Spectrometer to those from the Inner Detector, and requiring isolations from other tracks and energy deposits in calorimeters;

- jets: identified as energy deposits in both electromagnetic and hadronic calorimeters; the energetic clusters are processed by the antiKt jet reconstruction algorithm [7], with cone sizes $\Delta R=0.4$ or $\Delta R=0.6$. The antiKt algorithm is used because it is stable, fast, infrared- and collinear-safe. A calibration factor dependent on the cluster $p_{T}$ and $\eta$ is applied to correct for detector and physics effects [8]. The jet energy and direction is obtained from the ensemble of calorimetric clusters of energy associated with the jet;

- missing transverse energy: the $E_{T}^{\text {miss }}$ is a complex object which describes the unbalanced transverse momentum in each event; it represents a vector lying in the transverse plan and it is sensitive to the presence of neutrinos; the $E_{T}^{\text {miss }}$ depends also on the pile-up conditions and on the calibrations and resolutions of the Physics objects described above. Some analyses use the axial- $E_{T}^{\text {miss }}$ when the neutrino production is balanced on the transverse plane by the presence of other particles (see for instance Section 11). The axial- $E_{T}^{\text {miss }}$ is defined as the projection of the missing transverse momentum along the direction opposite to the recoil particles in the transverse plane. The $W W \longrightarrow l v l v$ analysis (Section 7) makes use of a modified missing transverse energy, $E_{T \text {, rel }}^{\text {miss }}$, defined as:

$$
E_{T, \text { rel }}^{\text {miss }}= \begin{cases}E_{T}^{\text {miss }} \times \sin (\Delta \phi) & \text { if } \Delta \phi<\pi / 2 \\ E_{T}^{\text {miss }} & \text { if } \Delta \phi \geq \pi / 2\end{cases}
$$

where $\Delta \phi$ is the difference in the azimuthal angle between the $E_{T}^{\text {miss }}$ and the nearest lepton or jet. This variable is designed to reject events when an apparent $E_{T}^{\text {miss }}$ arises from a mismeasurement of a lepton or a jet.

\section{Cross section measurement and common topics}

The diboson final state measurements do depend on the quality of the reconstructed objects as well as on the sub-detector resolutions. All the presented measurements have leptons in their final states, plus other objects as photons, jets or $E_{T}^{\text {miss }}$. As a consequence, the boson+jets and the $\bar{t} \bar{t}$ event categories are always present among the background processes: a jet can fake an electron or a gamma, or may be selected in hadronic final states, while the top contribution is important because it provides real $\mathrm{Ws}$ and jets. Other background contributions come from multijet events and pile-up.

The selections discussed in the following review have been introduced to decrease the contributions of the backgrounds in the various analyses without deteriorating the efficiency of the signal selection. 
In the following, six different diboson cross section measurements are briefly described, with a focus on the differences of the final states, backgrounds and cut applied. The references to the articles explaining the specific measurements are reported.

A summary of the results and limits on aTGC, as well as a comparison with the Standard Model predictions, is given in Section 12.

\section{$6 W \gamma / Z \gamma$ leptonic channels}

In this measurement a massive boson is produced in association with a high energy photon [9]. The diboson candidate events are selected requiring a lepton, $E_{T}^{\text {miss }}$ and a photon, or two opposite flavor leptons and a photon, or large missing transverse energy and a photon; these final states include also the photon bremsstrahlung from the leptons for the $W / Z$ decays. Events with massive bosons are identified with cuts on the transverse mass (for $W$ ) or on the invariant mass $(Z)$ of the leptonic final states, and an isolated $\gamma$ is requested, with a large angular separation from the leptons; the most important cuts for the various channels are listed in Table 2.

\begin{tabular}{|l||l|}
\hline process & cuts \\
\hline \multirow{4}{*}{$W \gamma \longrightarrow l v \gamma$} & an isolated lepton with $p_{T}^{l}>25 \mathrm{GeV}$ \\
& $E_{T}^{\text {miss }}>35 \mathrm{GeV}$ \\
& $M_{T}(W)>40 \mathrm{GeV}$ \\
& $p_{T}^{\gamma}>15 \mathrm{GeV}$ \\
& $\Delta R(\gamma, l)>0.7$ \\
\hline & two isolated-opposite charge leptons with $p_{T}^{l}>20 \mathrm{GeV}$ \\
$Z \gamma \longrightarrow l^{+} l^{-} \gamma$ & $M_{l l}>40 \mathrm{GeV}$ \\
& $p_{T}^{\gamma}>15 \mathrm{GeV}$ \\
& $\Delta R(\gamma, l)>0.7$ \\
\hline \multirow{3}{*}{$Z \gamma \longrightarrow v \bar{v} \gamma$} & $E_{T}^{\text {miss }}>90 \mathrm{GeV}$ \\
& $p_{T}^{\gamma}>100 \mathrm{GeV}$ \\
& $\Delta R(\gamma, l)>0.7$ \\
\hline
\end{tabular}

Table 2. List of cuts applied for the three channels of the $W \gamma / Z \gamma$ leptonic decays.

Figure 1 shows the signal differential cross section at particle level (where the detector effects have been unfolded) as a function of the photon transverse momentum, for data and Monte Carlo. In general, quite a good agreement is found between data and both the parton-shower (SHERPA [10] and ALPGEN [11]) and the Next to Leading Order genetator (MCFM [12]) calculations, the only disagreement being at large $E_{T}^{\gamma}$ for MCFM. This is due to the fact that the MCFM calculation used are up to 1 jet, and generally the high $p_{T}$ region is not very well described. The measurements do not highlight any deviations from the Standard Model predictions for the $V \gamma$ production cross section.

\section{$7 W W \longrightarrow l v l v$}

The $W W \longrightarrow l v l v$ signal is measured in final states with two opposite charge leptons and a large missing transverse energy [13]; depending on the lepton flavors, this analysis is split in three categories: $e e, \mu \mu, e \mu$. The background discrimination and estimation is challenging as several processes may fake this final state: real leptons and $E_{T}^{\text {miss }}$ in top events, fake $E_{T}^{\text {miss }}$ in Drell Yan processes, or jets 

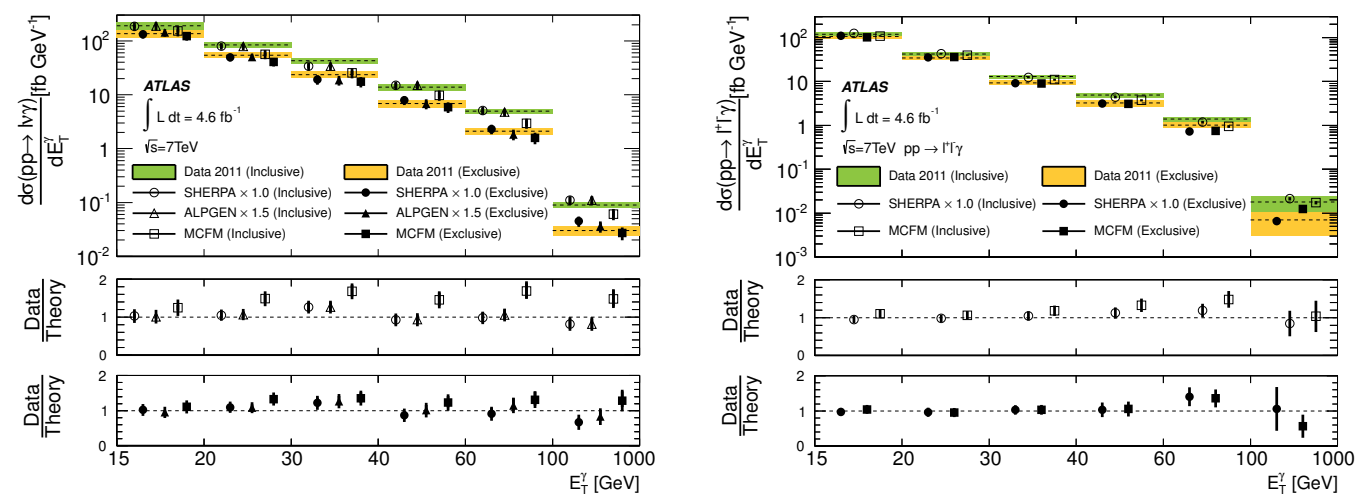

Figure 1. $E_{T}^{\gamma}$ differential cross sections for the $l v \gamma$ final state (left) and the $l^{+} l^{-} \gamma$ process (right plot) [9]. The exclusive cross section measurements in yellow have been obtained vetoing events containing any jets with $p_{T}>30 \mathrm{GeV}$; the inclusive measurement does not require any veto. The lower plots show the ratio of the data to different generators; the empty circles, triangles and boxes (top plots of the ratio panels) refer to the inclusive measurements, the full symbols refer to the exclusive selection (bottom plots of the ratio panels).

misidentified as leptons in $W+$ jets events. To suppress these backgrounds, different sets of cuts have been introduced:
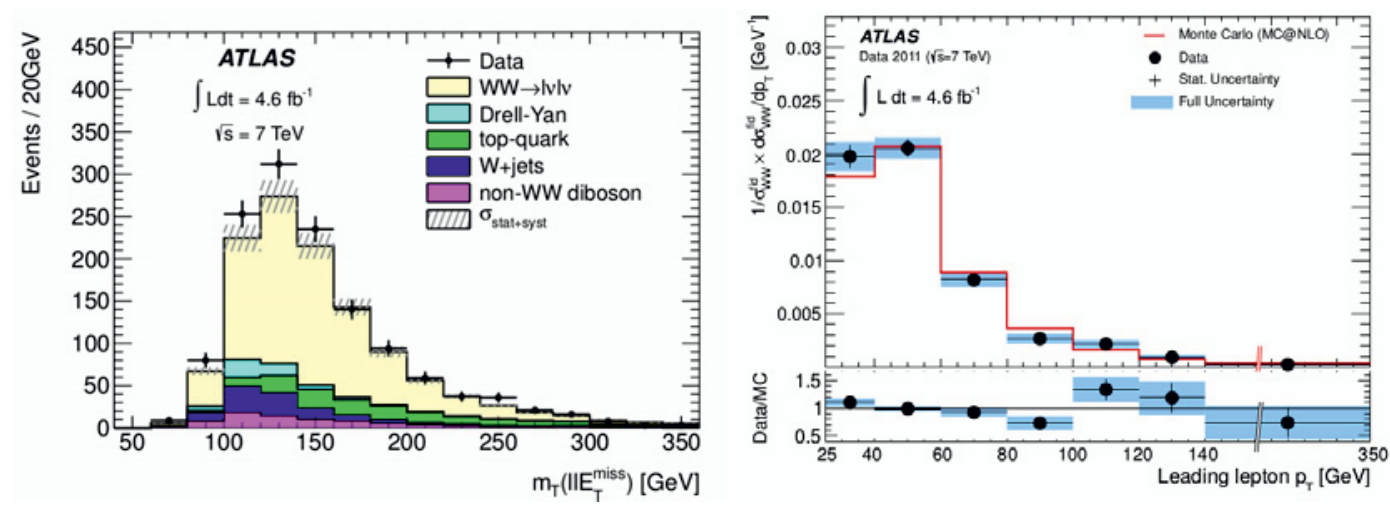

Figure 2. Left: distribution of the transverse mass of the system $\left(l^{\prime} l E_{T}^{\text {miss }}\right)$ system for $W W$ candidates with all selection criteria applied and after combination of $e e, \mu \mu$ and $e \mu$ channels, for both data (points) and Monte Carlo (histogram). Right: differential cross section for $W W \longrightarrow l v l v$ process as a function of the leading lepton transverse momentum for data (full circles) and Monte Carlo (histograms). Figures from [13].

- $\left|M_{l l}-M_{Z}\right|<15 \mathrm{GeV}$ and and to suppress Drell-Yan background in the ee and $\mu \mu$ categories;

- jet veto (to reject top background);

- $p_{T}(l l)>30 \mathrm{GeV}$ (suppress $W+$ jets background);

- $E_{T \text {, rel }}^{\text {miss }}>45 \mathrm{GeV}, 45 \mathrm{GeV}$ and $25 \mathrm{GeV}$ (to reject Drell-Yan processes) in the $e e, \mu \mu$ and $e \mu$ categories respectively; 
- lepton isolation and identification criteria (to reject $W+$ jets events).

Figure 2-left shows the transverse mass of the dilepton and $E_{T}^{\text {miss }}$ system after detector effect unfolding: the three main contributions to the background are the top, $W+$ jets and Drell-Yan processes. Figure 2-right shows the agreement between data and Monte Carlo for the leading lepton transverse momentum after the unfolding. The agreement between data and Standard Model prediction is within the uncertainties.

\section{$8 \mathrm{WZ} \longrightarrow l \mathrm{vll}$}

The $W Z \longrightarrow l v l l$ events are selected requiring three leptons and $E_{T}^{\text {miss }}$ in the final state, with two of the leptons being of the same flavor, opposite charge and compatible with an on-shell $Z$ boson [14]. The

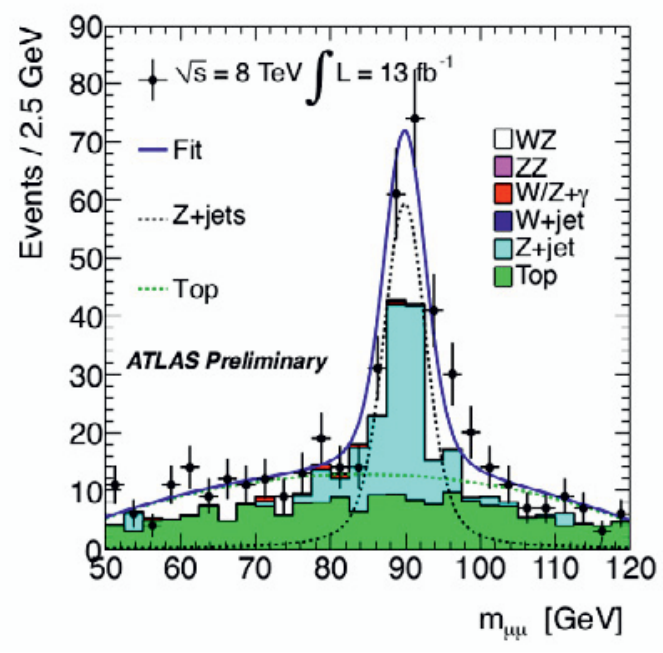

Figure 3. $M_{\mu \mu}$ distribution after the signal selection [14]. It is clearly visible the flat contribution from the top (green dots) and the peak of the $Z$ boson from the $Z+$ jets contribution. The blue line is the result of a fit to the data.

expected background for this channel are $Z+$ jets and top processes, with a jet misidentified as lepton, and $Z Z \longrightarrow$ llll. Figure 3 shows the di-muon invariant mass after the signal selection, comparing data (full circles) to Monte Carlo (histograms): the presence of three leptons in the final states reduces most of the backgrounds, making possible to relax the lepton $p_{T}$ cuts with respect to the other diboson analyses. In summary, the most important selection cuts applied are:

- 3 prompt leptons, $p_{T}^{l}>15 \mathrm{GeV}$

- lepton from the $W: p_{T}>20 \mathrm{GeV}$

- $\left|M_{l l}-M_{Z}\right|>10 \mathrm{GeV}$

- $E_{T}^{\mathrm{miss}}>25 \mathrm{GeV}$

- $M_{T}(W)>20 \mathrm{GeV}$

Figure 3 also shows the contributions to the background due to the top (green dots) and the peak of the $Z$ from $Z+$ jets events (black dots) at detector level (without unfolding); the blue line is the result of the fit to the data using these two components. 


\section{$9 W W / W Z \longrightarrow l v j j$}

The semileptonic diboson final state is the only measurement presented in this report with a pair of jets generated by the hadronic decay of a boson [15]. It tests the ability of the experiment to reconstruct dijet invariant masses in an environment in which the cross section for the jet production is very high. The most important backgrounds for this channel are the $W+$ jets and the $Z+$ jets processes. Final states with a top quark contribute to the background with a real $W$ boson, but they can be reduced with a jet multiplicity cut. The candidate jets are ordered in $p_{T}$ and only the leading and the subleading jets are considered to construct the boson mass.
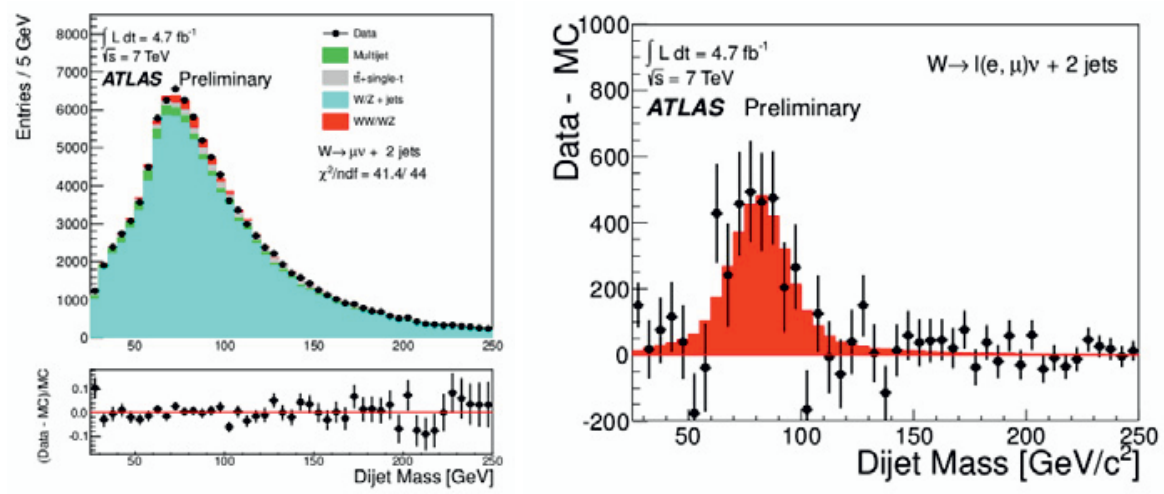

Figure 4. Left: dijet invariant mass distribution after the signal selection for both data and Monte Carlo in the muon channel. The diboson Monte Carlo signal is visible in red on top of the other backgrounds. Right: background-subtracted data after the combined fit in the electron and muon channels (full circles); the comparison with the signal yield from Monte Carlo is described by the histogram in red. Figures are from [15].

- single lepton (electron or muon) with $p_{T}>25 \mathrm{GeV}$

- $E_{T}^{\mathrm{miss}}>30 \mathrm{GeV}$

- $M_{T}(W)>40 \mathrm{GeV}$

- $p_{T}^{\text {lead jet }}>30 \mathrm{GeV}, p_{T}^{\text {sublead jet }}>25 \mathrm{GeV}$

- $\Delta \phi\left(E_{T}^{\text {miss }}, j^{\text {lead }}\right)>0.8$

Figure 4-left shows the dijet distributions at detector level for data and Monte Carlo, with highlighted the various contributions to the background. Figure 4-right shows the background subtracted data after a combined fit in the electron and muon channel: data and Monte Carlo agree within the errors and an eccess of data compatible with the signal is visible. The agreement with the Standard Model prediction is within the uncertainties.

\section{$10 \mathrm{ZZ} \longrightarrow 4 l$}

The purely leptonic decay of the $Z Z$ diboson system is reconstructed requesting the presence of 4 leptons in the final state [16]. This channel is the major background to the $H \longrightarrow Z Z \longrightarrow 4 l$ final state.

As in the case of the $W Z \longrightarrow l v l l$ channel, the presence of more than 2 leptons in the final state, with the request of same flavor-opposite charge, reduces considerably the background contributions, 
which are mainly due to the presence of a jet faking a lepton. Figure 5 shows the distribution of the leading lepton pair transverse momentum, before unfolding of the detector effects, both for data (full circles) and Monte Carlo (histogram).

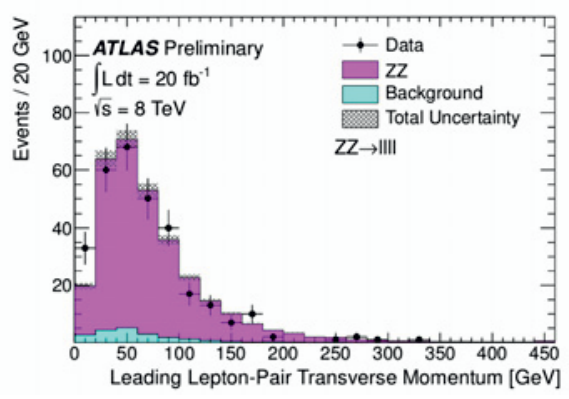

Figure 5. Distribution of the transverse momentum of the leading lepton pair in $Z Z \longrightarrow 4 l$ after signal selection, for data and Monte Carlo [16]; the cyan contribution is the Monte Carlo estimate of the background.

- 2 pairs of opposite sign leptons, $p_{T}^{l}>7 \mathrm{GeV}$

- $p_{T}>25 \mathrm{GeV}$ for the leading lepton

- $66 \mathrm{GeV}<M_{Z}<116 \mathrm{GeV}$

Data and Monte Carlo agree quite well within the errors.

\section{$11 \mathrm{ZZ} \longrightarrow l l v v$}

This is the second channel investigated in which a pair of $Z$ bosons is present in the final state, decaying to a pair of leptons and neutrinos [17]. For this analysis, the axial- $E_{T}^{\text {miss }}$ variable (defined as the projection of the missing transverse momentum along the direction opposite to the $Z \rightarrow l^{+} l^{-}$candidate in the transverse plane) is used instead of the $E_{T}^{\text {miss }}$, and the $Z$ decaying into leptons is requested to be balanced in the transverse plane by the $Z$ decaying in neutrinos:

- two opposite sign leptons with $p_{T}>20 \mathrm{GeV}$

- axial- $E_{T}^{\mathrm{miss}}>75 \mathrm{GeV}$

- $\left|E_{T}^{\text {miss }}-p_{T}(Z)\right| / p_{T}(Z)<0.4$

Figure 6 shows the unfolded differential $Z Z$ cross section as a function of $p_{T}(Z)$ : data and Monte Carlo are compared and no significant deviation is found with respect to the Standard Model prediction.

The results from this channel have been combined with those from the $Z Z^{*} \longrightarrow 4 l$ measurement; this channel has been investigated at the same $\sqrt{s}$ energy and requiring only one of the $Z$ bosons to be produced on-shell.

\section{Comparison with Standard Model predictions}

Table 3 collects the cross section results obtained for the presented channels, reporting the statistical, systematic and luminosity related uncertainties, for the fiducial cross sections, and the total cross sections when possible. A comparison with the expected results from NLO calculation in the Standard 


\section{ICNFP 2013}

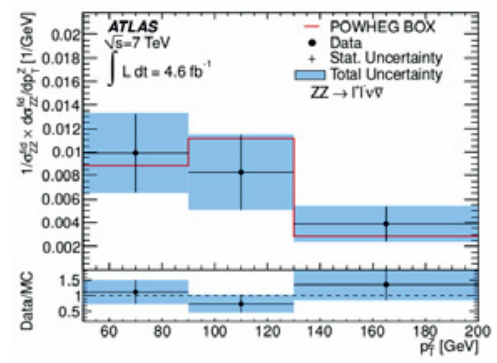

Figure 6. $p_{T}^{Z}$ differential cross section as a function of the di-lepton pair transverse momentum, for data and Monte Carlo [17]. The Monte Carlo describes the data within the errors.

\begin{tabular}{|c|c|c|c|c|c|}
\hline \multicolumn{2}{|l|}{ Process } & $\sqrt{s}$ & $\sigma_{\text {fid }}[\mathrm{pb}]$ (stat.) (syst.) (lumi.) & $\sigma_{\text {tot }}[\mathrm{pb}]$ (stat.)(syst.)(lumi.) & ref. \\
\hline$W \gamma / Z \gamma\left(N^{\mathrm{jet}}=0\right)$ & $\begin{array}{c}e v \gamma \\
\mu v \gamma \\
e^{+} e^{-} \gamma \\
\mu^{+} \mu^{-} \gamma \\
v \bar{v} \gamma\end{array}$ & $7 \mathrm{TeV}$ & see reference & $\begin{array}{c}1.77 \pm 0.04 \pm 0.24 \pm 0.08 \\
1.74 \pm 0.04 \pm 0.22 \pm 0.08 \\
1.07 \pm 0.03 \pm 0.12 \pm 0.04 \\
1.04 \pm 0.03 \pm 0.10 \pm 0.04 \\
(116 \pm 10 \pm 13 \pm 4) \cdot 10^{-3}\end{array}$ & [9] \\
\hline$W W \rightarrow l v l v$ & $\begin{array}{l}e e \\
\mu \mu \\
e \mu\end{array}$ & $7 \mathrm{TeV}$ & $\begin{array}{c}(56.4 \pm 6.8 \pm 9.8 \pm 2.2) \cdot 10^{-3} \\
(73.9 \pm 5.9 \pm 6.9 \pm 2.9) \cdot 10^{-3} \\
(262.3 \pm 12.3 \pm 20.7 \pm 10.2) \cdot 10^{-3}\end{array}$ & $\begin{array}{c}46.9 \pm 5.7 \pm 8.2 \pm 1.8 \\
56.7 \pm 4.5 \pm 5.5 \pm 2.2 \\
51.1 \pm 2.4 \pm 4.2 \pm 2.0\end{array}$ & [13] \\
\hline$W Z \rightarrow l v l l$ & & $8 \mathrm{TeV}$ & see reference & $20.3_{-0.7-1.1-0.6}^{+0.8+1.2+0.7}$ & [14] \\
\hline$W W / W Z \rightarrow l v j j$ & & $7 \mathrm{TeV}$ & see reference & $72 \pm 9 \pm 15 \pm 13$ & [15] \\
\hline$Z Z \rightarrow 4 l$ & $\begin{array}{c}4 e \\
4 \mu \\
2 e 2 \mu\end{array}$ & $8 \mathrm{TeV}$ & $\begin{array}{c}\left(4.6_{-0.7}^{+0.8} \pm 0.4 \pm 0.1\right) \cdot 10^{-3} \\
\left(5.0_{-0.5}^{+0.6} \pm 0.2 \pm 0.1\right) \cdot 10^{-3} \\
\left(11.1_{-0.9}^{+1.0} \pm 0.5 \pm 0.3\right) \cdot 10^{-3}\end{array}$ & $7.1_{-0.4}^{+0.5} \pm 0.3 \pm 0.2$ & [16] \\
\hline$Z Z \rightarrow l l v v, Z Z^{*} \rightarrow$ & & $7 \mathrm{TeV}$ & see reference & $6.7 \pm 0.7_{-0.3}^{+0.4} \pm 0.3$ & [17] \\
\hline
\end{tabular}

Table 3. Summary of the main cross section results for the presented measurements. For each measurement, the sub-channels are presented, as long as the center of mass energy, fiducial and total cross sections, and references. Refer to Table 1 for the integrated luminosities and data-taking conditions.

Model framework is presented in the references, and no deviation has been reported. Refer to the references for details on comparing the Standard Model predictions with the results in sub-channels and fiducial/total cross section regions, and for descriptions of uncertainty sources and their treatment. For the $W \gamma / Z \gamma$ measurement, the jet veto result is reported; see reference [9] for the inclusive measurement. The $W W / W Z \rightarrow l v j j$ analysis fits the electron and muon channel together, therefore there is no separated result. At the time of these proceedings, there is still discusion on how to present the fiducial cross section, since the the $\mathrm{W}$ and the $\mathrm{Z}$ cannot be resolved in the dijet invariant mass distribution (Figure 4-left), and the errors are large. The $Z Z \rightarrow l l v v$ reported results have been obtained combining with the $Z Z^{*} \rightarrow 4 l$ measurement; see the reference [17] for details on combinations and definitions of the acceptance. Figure 7 shows a comparison of the diboson cross sections as measured in different experiments and colliders as a function of the center-of-mass energy, while Figures 8 show a survey of the anomalous triple gauge couplings as measured by ATLAS and other experiments.

It can be seen that the precision of these measurements is really high, all the results confirm the validity of the Standard Model: in particular, all the tri-neutral coupling parameters are compatible to zero. For the definition and description of all the parameters reported in Figures 8 and 9 for the aTGCs, see [9] and references therein. 


\section{EPJ Web of Conferences}

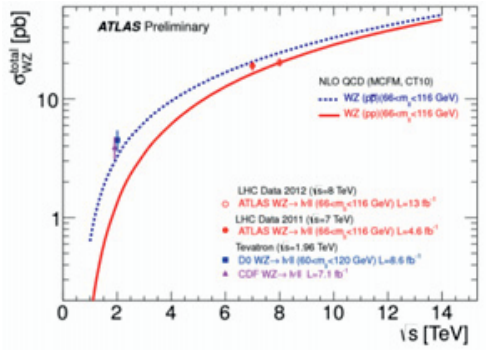

$W Z$

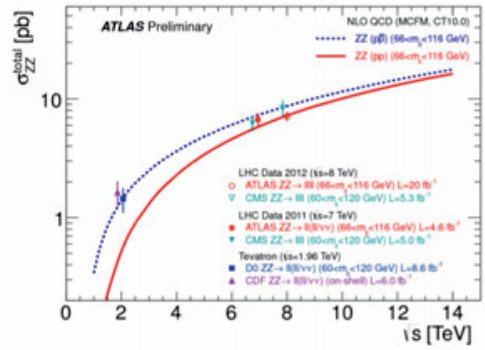

$Z Z$

Figure 7. Total production cross sections for $W Z$ on the left [14] and $Z Z$ diboson on the right [16] as a function of $\sqrt{s}$; experimental data are from Tevatron and LHC experiments.
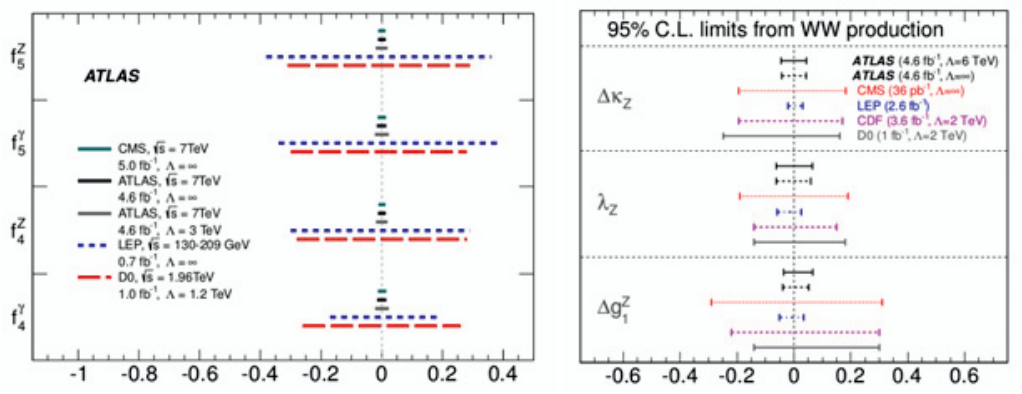

Figure 8. Summary of anomaluos Triple Gauge Couplings results from ATLAS and other experiments from LHC, Tevatron and LEP data, for ZZV (plot on the left, [17]) and WWV (plot on the right, [13]) couplings, where $\mathrm{V}=\mathrm{Z}$ or $\gamma$. The data show no disagreement with the Standard Model predictions.

\begin{tabular}{ccc}
\hline & Measured & Expected \\
processes & \multicolumn{2}{c}{$p p \rightarrow \ell \nu \gamma$} \\
\hline$\Lambda$ & $\infty$ & $\infty$ \\
$\Delta \kappa_{\gamma}$ & $(-0.41,0.46)$ & $(-0.38,0.43)$ \\
$\lambda_{\gamma}$ & $(-0.065,0.061)$ & $(-0.060,0.056)$ \\
\hline$\Lambda$ & $6 \mathrm{TeV}$ & $6 \mathrm{TeV}$ \\
$\Delta \kappa_{\gamma}$ & $(-0.41,0.47)$ & $(-0.38,0.43)$ \\
$\lambda_{\gamma}$ & $(-0.068,0.063)$ & $(-0.063,0.059)$ \\
\hline processes & $p p \rightarrow \nu \nu \gamma$ and & $p p \rightarrow \ell^{+} \ell^{-} \gamma$ \\
\hline$\Lambda$ & $\infty$ & $\infty$ \\
$h_{3}^{\gamma}$ & $(-0.015,0.016)$ & $(-0.017,0.018)$ \\
$h_{3}^{Z}$ & $(-0.013,0.014)$ & $(-0.015,0.016)$ \\
$h_{4}^{\gamma}$ & $(-0.000094,0.000092)$ & $(-0.00010,0.00010)$ \\
$h_{4}^{2}$ & $(-0.000087,0.000087)$ & $(-0.000097,0.000097)$ \\
\hline$\Lambda$ & $3 \mathrm{TeV}$ & $3 \mathrm{TeV}$ \\
$h_{3}^{\gamma}$ & $(-0.023,0.024)$ & $(-0.027,0.028)$ \\
$h_{3}^{Z}$ & $(-0.018,0.020)$ & $(-0.022,0.024)$ \\
$h_{4}^{\gamma}$ & $(-0.00037,0.00036)$ & $(-0.00043,0.00042)$ \\
$h_{4}^{2}$ & $(-0.00031,0.00031)$ & $(-0.00037,0.00036)$ \\
\hline
\end{tabular}

Figure 9. Measured and expected 95\% CL intervals on charged $\left(\Delta \kappa_{\gamma}\right.$ and $\left.\lambda_{\gamma}\right)$ and neutral $\left(h_{3}^{\gamma}, h_{3}^{Z}, h_{4}^{\gamma}, h_{4}^{Z}\right)$ anomalous couplings obtained by $W \gamma / Z \gamma$ measurement. The $\Lambda$ parameter is introduced to avoid unitary violation at high energy. Table is from [9]. 


\section{Summary}

The results for six different diboson production and decay channels obtained with the ATLAS detector at the LHC have been discussed, highlighting the excellent performances of both the accelerator and the experiment, all the analyses being limited by systematic uncertainties. Figure 10 reports some of the diboson cross section measurements and offers a comparison with other processes which act as backgrounds to the diboson final states, spanning a range of more than three order of magnitude.

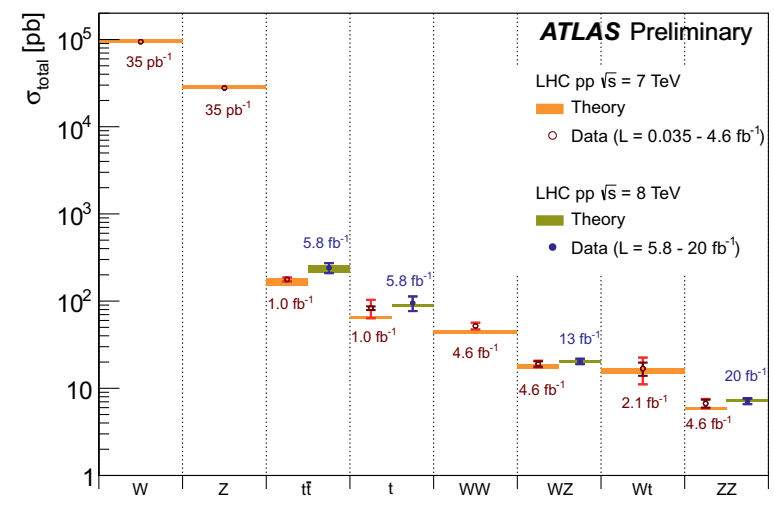

Figure 10. Summary plot of the Standard Model measurements in the Electroweak sector with the ATLAS detector using both 2011 and 2012 data [18]. The diboson tipical cross sections are about 3-4 order of magnitude smaller than the diboson background processes.

The measurements shows no significant deviations from the Standard Model predictions, and data and Monte Carlo agree quite well with NLO calculations.

The 6 diboson channels presented are sensible to different triple boson couplings, and can be used to set limits on the anomalous TGC; all the aTGC parameters, and in particular the triple-neutral boson couplings, are compatible with zero, but the sensitivity increases with the energy in the center of mass of the hard-interacting partons, $\sqrt{\hat{s}}$; the data taking campain foreseen after the long shutdown period will improve the results and allow the ATLAS experiment test the Standard Model at a higher energy scale.

\section{References}

[1] J. Beringer et al. (Particle Data Group), Phys. Rev. D86, 010001 (2012)

[2] The LEP-TGC combination group, http: lepww. web.cern.chlepwwtgc

[3] A. Robson, EPJ Web of Conferences 28, 06001 (2012).

[4] ATLAS Collaboration, JINST 3, S08003 (2008).

[5] ATLAS Collaboration, EPJ C (2013), 73:2518.

[6] https://twiki.cern.ch/twiki/bin/view/AtlasPublic/LuminosityPublicResults .

[7] M. Cacciari, G. P. Salam, G. Soyez, JHEP 0804:063 (2008).

[8] ATLAS Collaboration, ATLAS-CONF-2012-124, http://cds. cern. ch/record/1474490.

[9] ATLAS Collaboration, Phys Rev D 87, 112003 (2013).

[10] T. Gleisberg et al., JHEP 0902:007 (2009). 
EPJ Web of Conferences

[11] M. L. Mangano, F. Piccinini, A. Polosa, M. Moretti, and R. Pittau, JHEP 0307 (2003) 11.

[12] J. M. Campbell, R. Ellis and C. Williams, JHEP 1107, 018 (2011).

[13] ATLAS Collaboration, Phys Rev D 87, 112001 (2013).

[14] ATLAS Collaboration, ATLAS-CONF 2013-021, http://cds . cern . ch/record/1525557.

[15] ATLAS Collaboration, ATLAS-CONF 2012-157, http://cds . cern. ch/record/1493586.

[16] ATLAS Collaboration, ATLAS-CONF 2013-020, http://cds . cern. ch/record/1525555.

[17] ATLAS Collaboration, JHEP 03, 128 (2013).

[18] https://twiki.cern.ch/twiki/bin/view/AtlasPublic/StandardModelPublicResults. 